\title{
Kardiale Sarkoidose
}

\section{W. Schulte ${ }^{1}$ \\ U. Costabel ${ }^{1}$ \\ D. Kirsten ${ }^{2}$}

\section{Cardiac Sarcoidosis}

\section{Zusammenfassung}

Die Sarkoidose ist eine Systemerkrankung, bei der die frühzeitigen entzündlichen und unter Therapie rückbildungsfähigen Organveränderungen häufig subklinisch verlaufen. Bei narbigen Residuen ist oft keine Besserung mehr zu erreichen. Ein fortschreitender Funktionsverlust bis zum Organversagen kann auftreten. Der Beteiligung des Herzens kommt dabei eine wesentliche prognostische Bedeutung zu. Ein frühzeitiger Therapiebeginn mit Kortikosteroiden vor Auftreten irreversibler Funktionsstörungen des Herzens geht mit einer exzellenten Prognose einher. Die technischen Entwicklungen in den bildgebenden Verfahren haben zwar die grafische Darstellung von Struktur und Funktion des Herzens deutlich verbessert, dennoch ist bislang kein einzelnes Verfahren in der Früherkennung zuverlässig. Die Kombination verschiedener Verfahren und der klinischen Beschwerden erlaubt jedoch häufig eine frühe Diagnosestellung. Entscheidend ist das „daran denken“ und die rechtzeitige Einleitung einer weiterführenden Diagnostik bei entsprechenden Beschwerden. In der spezifischen Therapie fehlen weiterhin Alternativen zur Kortisontherapie. Die symptomatische kardiale Behandlung orientiert sich wesentlich an der Behandlung dilatativer Kardiomyopathien, da in vielen Frage wie z.B. der anti-arrhythmischen Therapie oder der Indikation zur Implantation antitachykarder Schrittmachersysteme keine, eigenständigen verlässlichen Studien zur Herzsarkoidose vorliegen.

\section{Abstract}

Sarcoidosis is a systemic disease, in which early inflammatory organic changes, which are often reversible under treatment, may take a subclinical course. In scarred residual tissue improvement is rare. Continuing functional loss or even organ failure is possible. The involvement of the heart is of essential prognostic value. Early onset of treatment with corticosteroids before irreversible functional loss of the heart occurs leads to an excellent prognosis. Technical progress in the imaging techniques have led to great improvement of the graphical presentation of cardiac structure and function, but not one of these techniques has yet been approved of for early diagnosis. Still, a combination of different diagnostical measures in addition to the evaluation of clinical symptoms often allows early diagnosis. A decisive factor is "to consider it" and a timely start of further diagnostics if certain symptoms are presented. Alternatives to the specific treatment with cortisone are still lacking. Symptomatic cardiac treatment is mainly oriented on the therapy of dilatatory cardiomyopathies, since there are no reliable systematic studies available on questions concerning, for example, antiarrhytmic treatment or indications for the implantations of pacemakers in connection with cardiac sarcoidosis.

${ }^{1}$ Abteilung Pneumologie/Allergologie, Ruhrlandklinik Essen

${ }^{2}$ Abteilung Pneumologie, Krankenhaus Großhansdorf.

Widmung

Prof. Dr. N. Konietzko zum 65. Geburtstag gewidmet.

Korrespondenzadresse

Dr. W. Schulte · Ruhrlandklinik · Tüschener Weg 40 · 45239 Essen · E-mail: schulte-adams@t-online.de

Eingang: 15. August $2003 \cdot$ Nach Revision alkzeptiert: 20. Oktober 2003

Bibliografie

Pneumologie 2003; 57: 752-760 @ Georg Thieme Verlag Stuttgart · New York · ISSN 0934-8387 
Die Sarkoidose ist eine Systemerkrankung, bei der nicht verkäsende Epitheloidzellgranulome jedes Organ befallen können. In den Frühstadien ist meist keine oder nur eine subklinische funktionelle Störung der betroffenen Organe vorhanden, ein ausgedehnter Befall führt jedoch bis zum Organversagen. Dem Herzbefall kommt dabei eine wesentliche prognostische Bedeutung zu. Da ein früher Therapiebeginn mit einer exzellenten Prognose einherzugehen scheint, ist die Diagnose der kardialen Sarkoidose vor Auftreten irreversibler Organschäden essenziell. Die Frühdiagnose ist jedoch auch heute noch „an imperfect science, a hesitant art“ (OP Sharma, Chest 2003). Wir möchten daher den aktuellen Stand in Diagnostik und Therapie der Herzsarkoidose darstellen.

\section{Epidemiologie}

Die Erstbeschreibung einer kardialen Sarkoidose erfolgte durch Bernstein u. Mitarb. 1929 [1]. Vier Jahre später wies auch Schaumann in zwei Autopsieberichten auf die Möglichkeit eines Herzbefalles hin [2]. Gentzen berichtete 1937 in seiner Veröffentlichung über Riesenzellgranulome bei zwei Fällen von Endokardfibrose über den ersten Todesfall, der einer Herzsarkoidose zugeschrieben wurde [3]. Auch in den weiteren Publikationen bis in die 80er-Jahre finden sich vielfach nur Kasuistiken, die teils zusammengefasst wurden.

Eine klinisch auffällige kardiale Beteiligung wurde anfänglich nur in einem geringen Anteil der Patienten (1 - 7\%) mit nachgewiesener Sarkoidose gefunden [4-7,10,13-18]. Bei diesen Patienten dürfte vor Einführung der Echokardiographie zusätzlich die Abgrenzung zum sekundären Cor pulmonale nur ungenau gewesen sein. Bei Anwendung moderner bildgebender Verfahren wie Echokardiographie, Szintigraphie, Magnetresonanztomographie etc. liegt der Anteil in der Regel höher, wird aber beeinflusst z.B. durch Selektion des Kollektivs oder die Besonderheiten der betreffenden medizinischen Versorgungsstrukturen [9,11,24-28]. Die höchsten Raten an Myokardbeteiligung mit 19,5 bis zu 78\% fanden Studien mit postmortaler histologischer Aufbereitung des Herzens [5, 6, 22, 29, 30 - 35] (Abb. 1 u. 2).

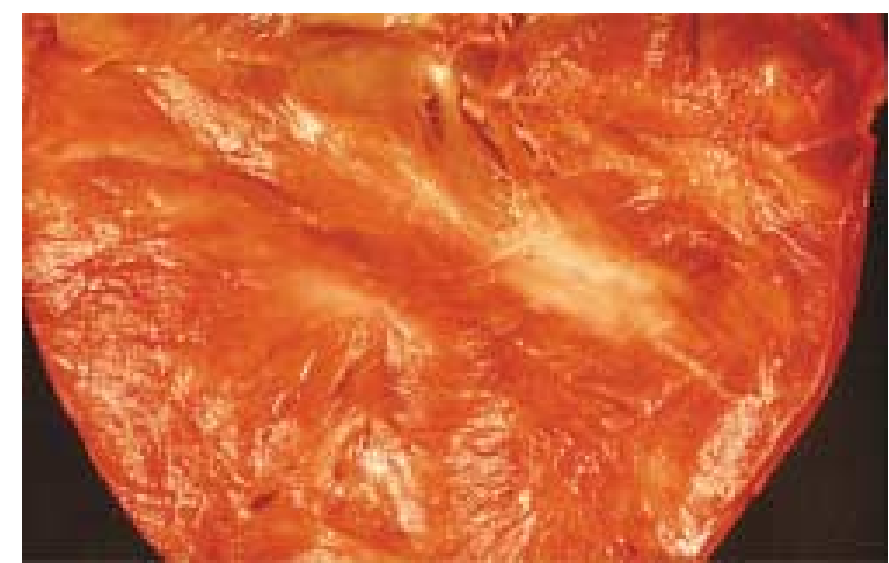

Abb. 1 Makroskopische Ansicht des linken Ventrikels eines an kardialer Sarkoidose verstorbenen Patienten.

1978 werteten Silverman u. Mitarb. 84 konsekutive Obduktionen von Patienten, die zwischen 1899 und 1977 am John-HopkinsHospital an einer Sarkoidose gestorben waren, aus. Sie fanden in $27 \%$ der Fälle eine Herzbeteiligung [30]. Ähnliche Zahlen berichteten Longscope und Freiman [32] und Sharma [5], die bei 92 bzw. 123 obduzierten Patienten in 20 bzw. 19,5\% histologisch eine Herzsarkoidose feststellten.

Scheinbar bestehen Unterschiede in der Krankheitsausprägung zwischen Patienten in Europa und Amerika im Vergleich zu Japan. Dort wurde aufgrund pathologischer Untersuchungen von einer Herzbeteiligung in $50-78 \%$ der Fälle berichtet $[31,34$, $38,39]$.

Diese wurde in Japan bislang zudem als führende Todesursache bei Sarkoidose angesehen. Während in den USA 13 - 50\% der Todesfälle auf eine kardiale Ursache zurückgeführt wurden, gelten in den japanischen Veröffentlichungen $77-85 \%$ als herzbedingt $[31,33,35,40]$.

In einer retrospektiven Studie aus Haifa/Israel verstarben dagegen zwischen 1980 und 1996 nur 2 von 120 Patienten mit Sarkoidose an einer kardialen Ursache [20].
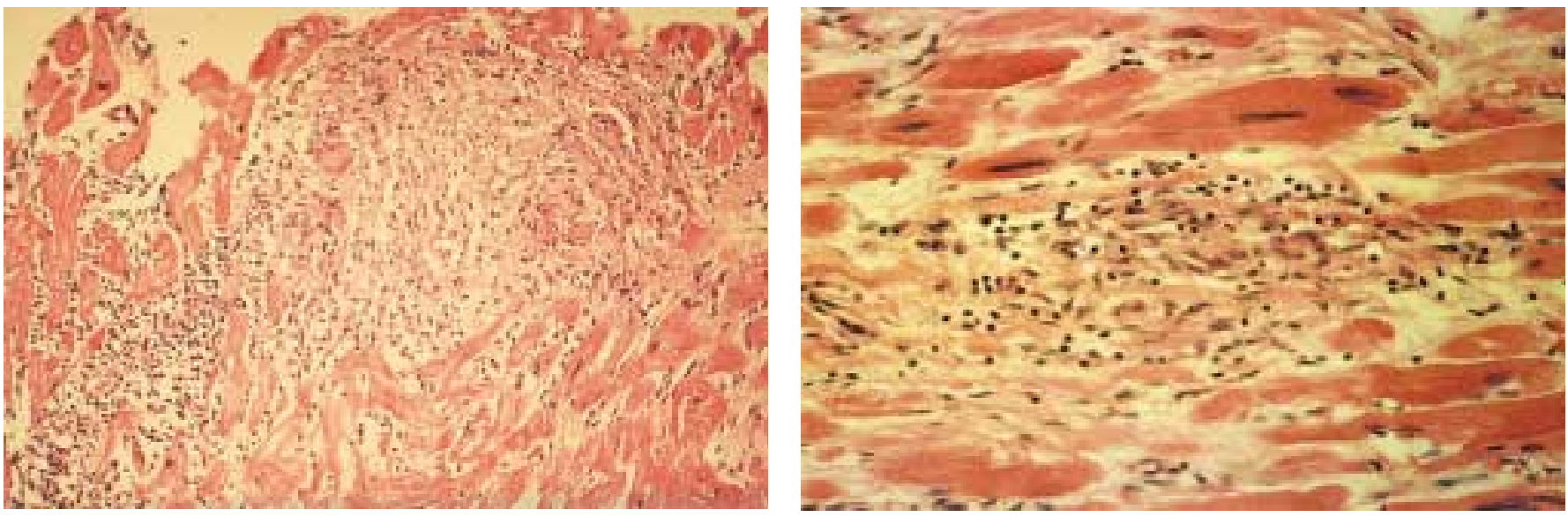

Abb. 2 a u. b Histologischer Befund mit intramyokardialen Sarkoidosegranulomen (HE-Färbung), a) bei schwacher, b) bei stärkerer Vergrößerung. 
Ebenfalls in einer retrospektiven Analyse des Krankengutes in Bad Berka fanden Kirsten u. Mitarb. eine Herzbeteiligung bei Patienten mit Lungensarkoidose in $15,1 \%$, eine prospektive Studie zur In-vivo-Diagnostik erbrachte einen Anteil von 10,6\%. Insgesamt zeigten 187 von 1236 Patienten mit Lungensarkoidose eine kardiale Beteiligung. Dabei wurden differenzialdiagnostisch infrage kommende Herzerkrankungen mittels Koronarangiographie und Herzbiopsie ausgeschlossen $[9,11]$.

Bei einer Befragung von 651 erkrankten Mitgliedern der Deutschen Sarkoidose-Vereinigung durch Kirsten 1992 gaben nur 8\% eine Herzbeteiligung an. Bei $47 \%$ der Patienten wurde eine extrapulmonale Organbeteiligung vermutet, in 30\% auch histologisch gesichert. Die Latenz zwischen Erstsymptom bzw. auffälligen Befunden bis zur Diagnosestellung betrug bis zu 7 Jahren, in der Nachsorge sei z. B. nur bei $51 \%$ ein Ruhe-EKG aufgezeichnet worden. Kirsten folgerte daraus, dass der systemische Charakter der Erkrankung in der Diagnostik wie Therapie häufig unterschätzt wird [10].

Die Beteiligung des Herzens verläuft häufig subklinisch, so dass insbesondere bei Patienten mit plötzlichem Herztod die kardiale Veränderung oft erst postmortem diagnostiziert wird $[7,12,22,29-31,33,36,37]$. Eine kardiale Sarkoidose scheint bei jungen Patienten häufiger zu sein und kann auch Heranwachsende betreffen [19,34-36].

In unserem Patientengut zeigen oft Patienten mit den schwergradigsten Herzveränderungen einen nur geringen pulmonalen und mediastinalen Befall. Hier erfolgte die Diagnose der Herzkomplikation meist durch den Kardiologen. Nur durch gezielte Untersuchungen wie BAL oder Mediastinoskopie bei geringen Veränderungen in der Computertomografie des Thorax konnte bei einzelnen Patienten auch eine pulmonale oder thorakale Erkrankung nachgewiesen werden. Andererseits besteht bei unseren Patienten mit ausgeprägtem pulmonalen Befall zwar in der Regel ein sekundäres Cor pulmonale, jedoch selten eine mit nicht invasiven Methoden nachgewiesene Myokardbeteiligung.

Auch Kirsten wies 1994 darauf hin, dass der Verlauf von Lungenund Herzsarkoidose nicht parallel verlaufen muss. So zeigten die Nachuntersuchungen in Bad Berka, dass in einem Drittel der Fälle die Herzsarkoidose erst dann klinisch auffällig wurde, nachdem die Lungensarkoidose sich radiologisch normalisiert hatte [21]. Daher sollte auch bei jungen Patienten mit unklaren Herzrhythmusstörungen oder Myokardveränderungen differenzialdiagnostisch an eine Sarkoidose gedacht werden.

\section{Klinik}

Bei nur mikroskopischem Nachweis einer Herzsarkoidose bleiben viele Patienten asymptomatisch. Bei stärkerem Befall können alle kardialen Strukturen befallen sein. Am häufigsten sind jedoch Veränderungen des Reizleitungssystems und des Myokards. Herzklappenfehler beruhen vornehmlich auf einer Beteiligung des betreffenden Papillarmuskels oder einer veränderten Ventrikelarchitektur. Ein direkter Befall der Herzklappen ist sehr selten.
Bei umschriebenen Veränderungen kommt es im akuten Stadium oft zu lokalen Hypertrophien oder begrenzten Bewegungsstörungen des Myokards, die im weiteren Verlauf durch Narbenbildung und Remodelling zu einer Erweiterung des Ventrikels bis hin zu Aneurysmen ( in 8-10\%) führen können. Ein diffuser Befall bedingt meist eine globale Hypokinesie bis hin zum kardialen Pumpversagen. Veränderungen des Reizleitungssystems und des Septums sind in der Regel mit bradykarden Herzrhythmusstörungen (AV-Block) oder Ausbildung eines Schenkelblockes verbunden. Auch bei klinischer Ausheilung verbleibt durch Narben ein arrhythmogenes Substrat, das z. B. über Mikro-Reentry-Tachyarrhythmien fördern kann. Im Bereich des Perikards sind Fibrosierungen und Perikardergüsse beschrieben. Entsprechend der Muskelmasse finden sich die Granulome am häufigsten im Septum und der freien Wand des linken Ventrikels und selten im Vorhofmyokard. Supraventrikuläre Herzrhythmusstörungen sind eher indirekte Folge einer ventrikulären Veränderung als Zeichen einer Infiltration der Vorhofwand [5,6,13, $14,22,23,29-31,41-46,48,53-55,87]$.

\section{Diagnostik}

\section{EKG}

Numao u. Mitarb. beobachteten bei 963 japanischen Patienten mit Sarkoidose in $22 \%$ Auffälligkeiten im Ruhe-EKG, fanden diese jedoch auch bei $17 \%$ der Vergleichsgruppe gesunder Probanden mit gleicher Alters- und Geschlechtsverteilung [47]. Bei 300 Patienten mit Herzsarkoidose in England wurden in $45 \%$ ventrikuläre Herzrhythmusstörungen, in 38\% Schenkelblockierungen, $28 \%$ supraventrikuläre Arrhythmien und 16\% ein plötzlicher Herztod beschrieben [6].

Die Prävalenz von EKG-Veränderungen scheint mit der Schwere der Herzbeteiligung zu korrelieren. Silverman u. Mitarb. verglichen 1978 klinische Befunde mit den Veränderungen in der Obduktion [30]. Dabei zeigten nur 15\% der Patienten ohne Herzbeteiligung Veränderungen im Ruhe-EKG, jedoch $42 \%$ der Patienten mit nur mikroskopisch nachweisbarer Beteiligung und 75\% der Patienten mit bereits makroskopisch sichtbarem Herzbefall.

Ein Vergleich schwedischer und japanischer Patienten durch Larsen u. Mitarb. 2001 ließ keine Unterschiede in der Häufigkeit und Art der EKG-Veränderungen erkennen [51].

Verstärkte oder abgeleitete EKG-Darstellungen wie Spätpotenziale oder Herzfrequenzvariabilität spielen weder in Diagnostik noch Prognoseabschätzung eine Rolle.

Belastungs-EKG und Spiroergometrie scheinen etwas sensitiver zu sein, spielen aber mehr in der Differenzialdiagnostik z. B. zur Ischämie oder bez. einer kardiovaskulären vs. pulmonalen Leistungslimitierung eine Rolle [5,49-52].

Dem Langzeit-EKG kommt sowohl in der Diagnostik als auch der Risikoabschätzung bei nachgewiesener Herzsarkoidose eine größere Rolle zu. Suzuki u. Mitarb. fanden in einer prospektiven Studie mit 38 Sarkoidosepatienten in $67 \%$ mehr als 100 ventrikuläre Extrasystolen pro Tag, falls eine Herzsarkoidose vorlag, jedoch nur in $8 \%$ der Fälle ohne Herzbeteiligung. Eine Vergleichsgruppe 
zeigte nur bei $5 \%$ von 58 gesunden Probanden mehr als 100 VES/ Tag 849].

\section{Echokardiographie}

Die Echokardiographie ist eine sehr hilfreiche Methode, die sowohl morphologische als auch funktionelle Veränderungen des Herzens mit hoher Auflösung und fehlender Belastung für den Patienten aufzuzeigen vermag. Bei geringen Kosten und hoher Verfügbarkeit ist sie zudem vorzüglich zur Verlaufsbeobachtung geeignet. Die Sensitivität lässt sich noch durch die Stress-Echokardiografie steigern.

Die Echokardiographie ist bei 14-41\% aller Sarkoidosepatienten pathologisch, teils bei normalem Ruhe-EKG [24,26,27,53-59, 69]. Viele Studien wurden jedoch in den 80er-Jahren durchgeführt, als die Ultraschalltechnik noch eine deutlich schlechtere Auflösung ermöglichte als die heutigen Geräte. Aus unserer Sicht kommt der Echokardiographie heute eine wesentliche Rolle in Diagnostik und Verlaufsbeobachtung zu.

1998 verglich Yazaki [101] 15 Patienten mit Herzsarkoidose und 30 Patienten mit dilatativer Kardiomyopathie (DCM). Von den Patienten mit nachgewiesener Herzsarkoidose zeigten $73 \%$ eine umschrieben verdickte oder verdünnte Wand des linken Ventrikels, in der Regel das Septum betreffend und dann immer mit einer AV-Blockierung einhergehend. Von den Patienten mit DCM besaßen nur 17\% umschriebene Wanderweiterungen, jedoch keine umschriebenen Hypertrophien. Auch weitere Erkrankungen wie primäre Vitien, Cor pulmonale, hypertrophe Kardiomyopathien oder Amyloidose sind wegen typischer oder pathognomonischer Merkmale echokardiographisch mit großer Sicherheit differenzierbar. Diastolische Funktionsstörungen werden für ca. $50 \%$ aller Patienten mit nachgewiesener Herzsarkoidose in den Studien zur Echokardiographie und übereinstimmend zur Magnetresonanztomografie berichtet $[71,139]$.

\section{Nuklearmedizinische Untersuchungen}

Am besten untersucht ist die Myokardszintigraphie mit 201Thallium. Dieses Radionuklid wird von den Herzmuskelzellen aufgenommen. Dabei reichern Bereiche mit Narben, Nekrosen und teils Entzündungen weniger Thallium an und erscheinen als „cold spots“. Die in Ruhe aufgezeichneten Defekte sind bei sarkoidalen Veränderungen in den Spätaufnahmen und unter Belastung rückläufig oder reversibel. Dieses Phänomen wird „reverse distribution“ genannt und erlaubt eine Abgrenzung zu ischämischen Veränderungen bei koronarer Herzkrankheit, die die häufigste und wichtigste Differenzialdiagnose bei umschriebenen Myokardveränderungen darstellt.

Da nur ein geringer Anteil der Sarkoidosepatienten eine relevante kardiale Beteiligung aufweist, ein pathologischer Befund bei asymptomatischen Patienten offenbar mit günstiger Prognose einhergeht und die Bedeutung dieser Konstellation für den weiteren Krankheitsverlauf und Therapie nicht gesichert ist, kommt dieser Untersuchung nicht die Stellung einer Routinemethode zu. Der Nachteil der Technik liegt in der hohen Strahlenbelastung. Dies schränkt die Anwendung der Methode in der Verlaufskontrolle ein. Dennoch ist bislang für keine andere Untersuchungstechnik eine bessere Sensitivität gesichert worden $[5,24,25,28,61-65,70]$.
Der Anteil pathologischer Befunde bei der Thalliumszintigraphie schwankt dabei zwischen 13 und 75\%, je nach Größe und Zusammensetzung des untersuchten Patientenkollektiv.

Die 67Gallium-Szintigraphie weist nach Studienlage eine geringere Sensitivität als die 201TI-Szintigraphie auf [68,73-78, $80,82,84]$. Patienten mit positivem Befund in der 67Ga-Szintigraphie zeigen fast immer auch Auffälligkeiten in der 201TISzintigrafie. Eine Kombination beider Verfahren zur Verbesserung der Diagnostik und Prognoseabschätzung wurde diskutiert, ist letztlich jedoch nicht gesichert. Auch eine Darstellung kardialer Veränderungen bei Herzsarkoidose mittels Technetium99-pyrophosphate wurde berichtet, es liegen jedoch keine vergleichenden Studien vor [54,81]. Untersuchungen mit 99m-Tc-sesta-methoxy-isobutyl-isonotrile (sestamibi) SPECT scan könnte der Darstellung mit Thallium überlegen sein, jedoch ist auch dies aufgrund der wenigen Studien letztlich nicht gesichert $[61,83]$.

\section{Magnetresonanztomographie (MRT)}

Die MRT wird zunehmend in der Diagnostik der Herzsarkoidose eingesetzt. Es liegen neben einzelnen Fallberichten seit 1988 bislang nur wenige größere Studien vor. Shimada fand bei kontrastverstärktem MRT mittels Gadolinium-DTPA bei 8 von 16 Patienten mit Herzsarkoidose umschriebene Kontrastverstärkungen wie bei interstitieller Ödembildung, Entzündung oder Vernarbung [70]. Bei hoher struktureller Auflösung sind morphologische und funktionelle Veränderungen gut darstellbar, aufgrund unterschiedlicher Verteilungsmuster ischämische Läsionen meist abgrenzbar (Abb. 3-5). Bei primär positivem Befund eignet sich die Methode daher sehr gut zur Verlaufsbeobachtung $[72,85,86]$. Unsere Erfahrungen zeigen relativ typische und deutliche Ergebnisse bei akuter, nicht vorbehandelter Herzsarkoidose. Andererseits fanden wir bei mehreren Patienten mit histologisch gesicherter Herzsarkoidose und mehrjährigem Verlauf unter einer Steroidtherapie zwar Veränderungen der Herzstruktur wie reduzierte systolische Funktion oder umschriebene Wandveränderungen, jedoch keine pathologischen oder lokalen Auffälligkeiten im Myokard selbst.

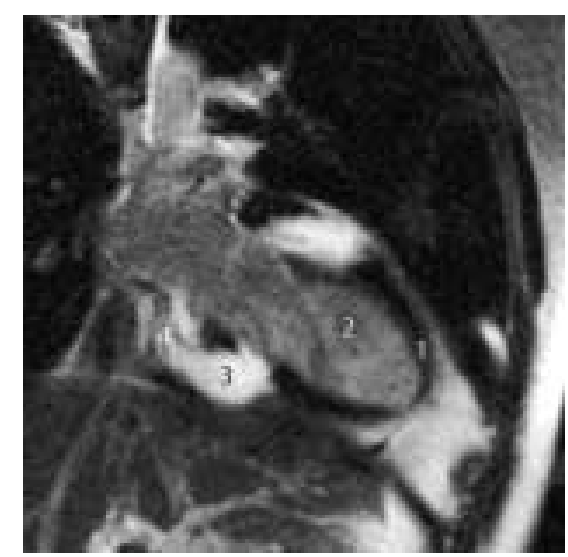

Abb. 3 Cardio-MRTBild eines Patienten mit gesicherter Herzsarkoidose. Weiß dargestellte Konstrastmittelanreicherung (Gadolinum) der akut befallenen Myokardanteile im Bereich der Papillarmuskelansätze in linken Ventrikel. Dunkel gefärbt zeigen sich die nicht betroffenen Myokardanteile und die in der Schnittebene liegenden Herzkranz-

gefäße (Zwei-Kammerblick des linken Ventrikel in langer Achse, 1,5 Tesla, T1-Gewichtung, fast spin echo, Kontrastmittel Gadolinum, Inversion recovery sequence). 1 . normal kontrastiertes Myokard (dunkel); 2. blutgefülltes Lumen des linken Ventrikels (grau); 3. kontrastmittelangereicherte Myokardanteile im Bereich der Papillarmuskeln des linken Ventrikels (hell); 4. Koronargefäße. 


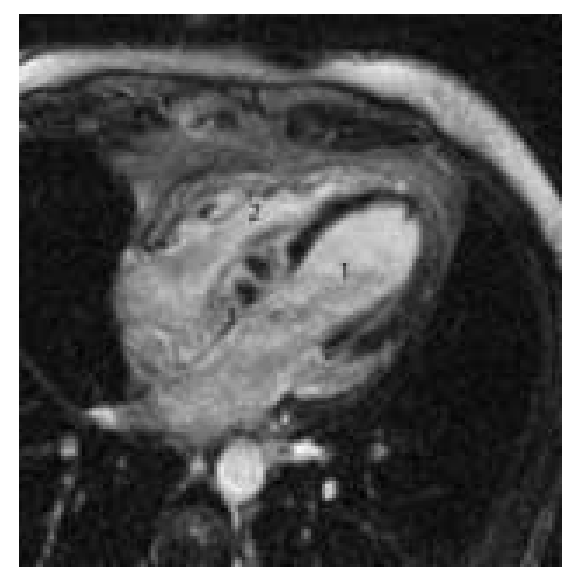

Abb. 4 Typische Verdickung und Kontrastierung des basalen Septums im Vierkammerblick des CardioMRT eines Patienten, bei dem ein AV-Block 2.-3. Grades die Diagnostik einleitete. 1. linker Ventrikel; 2. rechter Ventrikel; 3. verdicktes basales Septum mit inhomogener Kontrastmittelanreicherung.

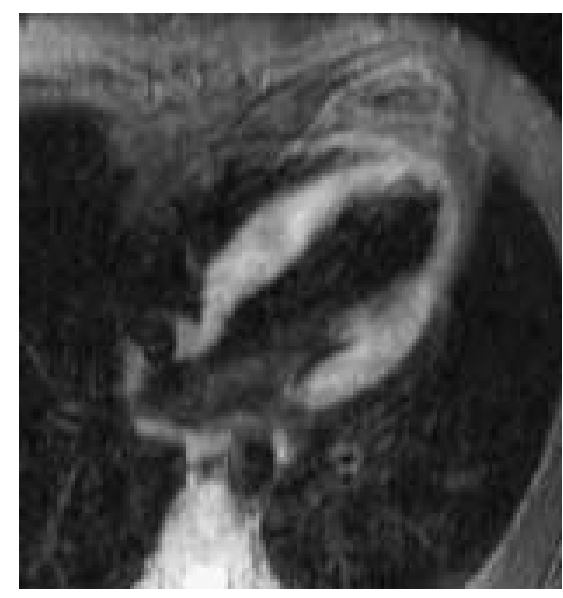

Abb. 5 Diffuser Befall des Herzens mit histologisch gesicherter Herzsarkoidose. Im MRT sind die herdförmigen Kontrastunterschiede und umschriebenen Verdickungen des Myokards gut erkennbar ( gleiche Sicht, jedoch veränderte T1/T2-Gewichtung wie Abb. 2, so dass sich das gesunde Myokard hell darstellt).

Unsere bisher nicht publizierten Daten aus der Ruhrlandklinik entsprechen den Ergebnissen von Skold u. Mitarb. mit hoher Rate unspezifischer Veränderungen in Echokardiografie und MRT, aber einer nur geringen Rate relativ spezifischer Befunde [71]. Zahlreiche Studien zu diesem Themenbereich sind in den nächsten Jahren zu erwarten. Durch weitere technische Entwicklungen mit Verbesserung der Auflösung könnte diese Methode auch wegen der geringen Belastung für den Patienten zunehmend an Bedeutung gewinnen und z.B. die nuklearmedizinischen Tests ablösen. Schrittmacherträgern bleibt die Untersuchung jedoch vorenthalten [70,71,79,85-94].

\section{Positronenemissionstomographie (PET)}

Bislang liegen nur Kasuistiken und eine kleine Studie mit $16 \mathrm{~Pa}-$ tienten vor $[95,96]$. Ob die PET für diese Indikationsstellung Bedeutung erlangen wird, ist bislang nicht abzuschätzen.

\section{Invasive Untersuchungen}

Die Koronarangiographie wird heute bei der Mehrzahl der Patienten mit Herzsarkoidose durchgeführt und dient dem Ausschluss einer Erkrankung der Koronararterien bei umschriebenen Veränderungen des Herzens oder im EKG.

Myokardbiopsien werden fast ausschließlich aus dem rechten Ventrikel entnommen, die meisten sarkoidalen Läsionen sind jedoch im Myokardbereich des linken Ventrikels verteilt. Je geringer und umschriebener die granulomatösen Infiltrate sind, desto geringer ist die Wahrscheinlichkeit, eben diesen Bezirk mit der
Biopsie auch zu treffen. Kommt es durch eine vorangegangene Behandlung zu einem Abheilen der Granulome und Ausbildung narbiger Veränderungen, so sind die histologischen Bilder trotz repräsentativer Probe ebenfalls nur begrenzt aussagekräftig. Die Erfolgsrate ist daher nur bei diffusem schweren Befall akzeptabel und liegt im Allgemeinen < 25\% [38,39,47,60,98-100]. Da zudem noch erhöhte Risiken und Belastungen für den Patienten bestehen, kann diese Methode nicht generell empfohlen werden.

\section{Klinische Diagnosekriterien}

Wir haben die 1993 vom „Japanese Ministry of Health and Welfare“ aufgestellten Kriterien zur Diagnose der Herzsarkoidose als sehr hilfreich und zuverlässig empfunden (siehe Tab.1). Hier wird zwischen histologisch gesicherter und klinischer Diagnosestellung unterschieden. Für die klinisch geleitete Diagnose wird die histologische Sicherung einer Sarkoidose extrakardial verlangt. Zusätzlich müssen neben definierten EKG-Veränderungen wie z.B. Schenkelblock, AV-Block oder komplexen ventrikulären Herzrhythmusstörungen auch strukturelle oder funktionelle Veränderungen des Myokards vorliegen. Ausgeschlossen sind jedoch Patienten, bei denen sich die Veränderungen bereits durch andere Ursachen wie Myokardinfarkt oder Cor pulmonale erklären.

Tab. 1 Guidelines for Diagnosing Cardiac Sarkoidosis, Japanese Ministry of Health and Welfare, 1993 [70].

1. Histologische Diagnostik
Nachweis eines nicht verkäsenden epitheloidzelligen Granuloms im Myokard
2. Klinische Diagnosestellung
Nach histologischem Nachweis einer extrakardialen Sarkoidose kann eine Herz-
sarkoidose angenommen werden, wenn ein Kriterium der Gruppe a und min-
destens ein weiteres Kriterium der Gruppe b-e vorliegt.
a. Kompletter RSB, Linkslagetyp, AV-Block, ventrikuläre Tachykardie, VES > Lown
2, abnormes Q, ST-Strecken- oder T-Wellenveränderungen
b. Abnorme Wandbewegungen, umschriebene Herzwandverdünnungen, Dilata-
tion des linken Ventrikels
c. Defekte in der Myokardszintigraphie
d. Abnormer intrakardialer Druck, low cardiac output, verminderte Ejektions-
fraktion
e. Interstitielle Fibrose oder mehr als mäßige Zellinfiltration in der Myokardbiop-
sie bei nicht spezifischem Befund.

\section{Beteiligung anderer Organsysteme}

Die wenigen publizierten Daten wie auch eigene Erfahrungen zeigen, dass fast immer eine Beteiligung der Lunge und/oder des Mediastinums vorliegt [9-11,13,29,42]. Yazaki [42] beschreibt bei 95 Patienten mit Herzsarkoidose in $41 \%$ eine zusätzliche Augen- und in $16 \%$ eine Hautbeteiligung. Fleming fand bei 20 obduzierten Patienten in 35\% eine zusätzliche Leber- und Milzinfiltration [13].

Kirsten fand bei 187 Patienten mit Herzsarkoidose einen Befall von Hilus-Lymphknoten in $94 \%$, der Lunge in $77 \%$, der Leber und Milz in 57 bzw. 50\% und weiterer Organsysteme in 37\% der Fälle [9,11]. Eine Assoziation der Herzsarkoidose mit Lungenfunktionsparametern, der Form oder des Schweregrades der Lungensarkoidose oder dem ACE-Spiegel sind nicht beschrieben. 
Die primäre Behandlung der Herzsarkoidose entspricht dem für Sarkoidose generell üblichen Vorgehen, d.h. sie besteht vornehmlich in der Gabe von Kortikosteroiden [5-7,14,70,97,98, 102 - 104,115]. Auch hier liegen zahlreiche Kasuistiken und kleinere retrospektive Auswertungen vor, jedoch kaum prospektiv randomisierte Studien.

Während einzelne Berichte von z.T. sehr gutem kurzfristigen Ansprechen auf Kortison berichten, ist die Datenlage zu langfristigem Verlauf, prognostischen Faktoren, Dauer und Höhe der Steroidtherapie sehr gering. Auch die Medikation bei kardialer Dysfunktion oder Herzrhythmusstörungen erfolgt nach empirischen oder theoretischen Überlegungen in Anlehnung an andere Kardiomyopathien, deren Therapie in großen Studien validiert wurde.

Während die Indikation zur Implantation eines antibradykarden Schrittmachersystems einfach zu stellen ist, herrscht bei der Indikation zu antitachykarden Schrittmachersystemen (AICD) Unsicherheit bez. des richtigen Zeitpunktes. Offen ist die Frage, ob sie nur zur Sekundärprävention nach Tachyarrhythmie bedingter Synkope/Reanimation eingesetzt werden sollten oder bereits zur Primärprävention bei als gefährdet betrachteten Patienten. Für letzteres Vorgehen würde die erwartete hohe Sterberate an plötzlichem Herztod sprechen. Auch der Einfluss der Therapie auf das Risiko des plötzlichen Herztodes ist ungewiss, da nach Umwandlung des entzündlichen Infiltrates in eine Narbe ein proarrhythmisches Substrat bestehen bleibt [102,127 - 132].

Eine sehr hilfreiche Studie, die sicher wesentlich zur Abschätzung dieser Fragen beigetragen hat, ist die Arbeit der „Central Japan Heart Study Group“ [42]. Sie werteten retrospektiv die Daten von 95 Patienten aus, bei denen zwischen 1984 und 1996 eine Herzsarkoidose diagnostiziert worden war. Nach einer durchschnittlichen Beobachtungszeit von 68 Monaten verstarben 29 Patienten ( $=73 \%$ ) an einem zunehmenden kardialen Pumpversagen und 11 Patienten $(=27 \%$ ) an plötzlichem Herztod. Die multivariante Analyse identifizierte den funktionellen NYHAStatus (hazard ratio 7,72 pro NYHA-Klasse, $p=0,0008$ ), den enddiastolischen Durchmesser des linken Ventrikels (hazard ratio 2,6 pro $10 \mathrm{~mm}$ Zunahme, $\mathrm{p}=0,02$ ) und anhaltende ventrikuläre Tachykardien (hazard ratio 7,2, p =0,03) als unabhängige Prädiktoren der Mortalität. Andererseits fanden sie eine exzellente Prognose bei den Patienten, bei denen nach früher Diagnose eine Therapie mit Steroiden erfolgte.

Die 5-Jahres-Überlebensrate betrug 75\% für alle Steroid-behandelten Patienten. Lag die Ejektionsfraktion (EF) des linken Ventrikels vor Therapiebeginn $>50 \%$, so bestand eine nahezu normale Lebenserwartung mit einer 10-Jahres-Überlebensrate von $89 \%$. Von den Patienten mit bereits reduzierter kardialer Funktion und einer $\mathrm{EF}<50 \%$ lebten dagegen nur noch $59 \%$ nach 5 Jahren und $27 \%$ nach 10 Jahren. Die Untersucher fanden keinen Unterschied zwischen den Patienten mit einer Steroiddosis kleiner versus größer $30 \mathrm{mg}$ Prednison/die. Für eine Gruppe von $20 \mathrm{~Pa}-$ tienten mit durch Obduktion gesicherter Erkrankung und nicht erfolgter Kortisonbehandlung wurde retrospektiv eine 5-JahresÜberlebensrate von nur $10 \%$ ermittelt.
Obwohl große prospektive Studien fehlen, kann man von der Wirksamkeit einer Steroidbehandlung ausgehen. Dennoch bleiben die Fragen zur Dosis und einem möglichen Therapieende ungeklärt. Wir haben bereits mehrfach nach weiterer Dosisreduktion unter $10 \mathrm{mg}$ Prednisolon trotz zuvor stabilem Verlauf eine erneute Verschlechterung gesehen, sodass wir bis zum Vorliegen weiterer Daten von einer vermutlich dauerhaft erforderlichen Behandlung ausgehen. Die Mindestdauer einer Kortisontherapie bei Herzsarkoidose betrug nach den Erfahrungen unserer Kliniken und dem Sarkoidosezentrum der früheren DDR in Bad Berka 2 Jahre.

Als zusätzliche Medikation liegen Einzelberichte für Methotrexat, Azathioprin, Hydrochlorothiazid, Cyclophosphamid, Cyclosporin A, Thalidomid, Pentoxifyllin, Infliximab (Anti-Tumor-Nekrose-Faktor) und Chloroquin vor. Außer einem kortisonsparendem Effekt der immunsuppressiven Medikamente konnte jedoch bislang keine Wirksamkeit dieser Medikamente bez. der Herzsarkoidose gesichert werden [103,105-126].

Bezüglich einer medikamentösen antiarrhythmischen Therapie liegen ebenfalls keine gesicherten Daten vor. Entsprechend der Datenlage für andere Kardiomyopathien wird bevorzugt Amiodaron eingesetzt. Auch die Aussagekraft elektrophysiologischer Untersuchungen scheint begrenzt [127-132].

Kleinere Studien bewiesen einen Überlebensvorteil bei Implantation eines AICD. So berichten Winters u. Mitarb. 1997 [60] von 7 Patienten mit Herzsarkoidose und dokumentierter anhaltender ventrikulärer Tachykardie. Trotz anti-arrhythmischer und steroidaler Therapie verstarben 2 Patienten an plötzlichem Herztod und weitere 4 Patienten zeigten Rezidive ventrikulärer Tachykardien. Nach Implantation eines AICD bei 4 Patienten wurde bei allen mindestens eine adäquate Schockabgabe dokumentiert.

Insgesamt scheint nach Ansicht mehrerer Autoren bereits die prophylaktische Implantation eines AICD bei besonders befährdeten Patienten gerechtfertigt zu sein $[60,90,128,132,133]$.

Bei den selten vorkommenden Perikardergüssen können Perikardpunktionen, Perikardfensterungen oder bei massiven Ergüssen Perikardektomien erforderlich sein $[23,43,53-55]$.

Zuletzt sollte bei den oft relativ jungen Patienten rechtzeitig die Möglichkeit einer Herztransplantation erörtert werden [7,101,136]. Ein Rezidiv der Sarkoidose im Herztransplantat ist möglich, über ein Ansprechen auf eine Erhöhung der Steroiddosis wurde berichtet $[134,135,138]$. Die Mortalität der Patienten mit Lungensarkoidose auf der Warteliste zur Transplantation entspricht den Patienten mit Lungenfibrose, wobei insbesondere die Rechtsherzbelastung zunehmende Probleme bereitet [137]. Bezüglich des Zeitpunktes zur Listung bei führender kardialer Sarkoidose liegen keine validen Daten vor, hier ist der vorangegangene Verlauf entscheidend. Insbesondere bei nicht vorbehandelten Patienten sollte aber immer ein Therapieversuch vorangehen, da einige Patienten unter Kortison erhebliche funktionelle Verbesserungen zeigen und so möglicherweise die Transplantation vermieden werden kann $[29,41,42,101]$. 


\section{Danksagung}

Wir danken den Kollegen der kardiologischen und radiologischen Abteilung des Elisabeth-Krankenhauses Essen ( Prof. Dr. G. Sabin, PD Dr. J. Barkhausen, Dr. O. Bruder, Dr. W. Schüler, Dr. Jochims ) für die freundliche Durchführung und Überlassung der MRT-Bilder.

Doz. Dr. V. Haenselt, Bad Berka, danken wir für die Überlassung der mikro- und makroskopischen Bilder der Herzsarkoidose.

\section{Literatur}

${ }^{1}$ Bernstein M, Konzelman FW, Sidlick DM. Boeck's sarcoid: report of a case with visceral involvement. Arch Intern Med 1929; 4: 721 - 734

${ }^{2}$ Schaumann J. Etude anatomo-pathologique et histologique sur les localisationes viscerates de la lymphogranulomatose benigne. Bull Soc franc Dermat Syph 1933; 40: 1167-1171

${ }^{3}$ Gentzen G. Über Riesenzellgranulome bei zwei Fällen von Endokardfibrose. Beiträge zur pathologischen Anatomie 1937; 98: 375

${ }^{4}$ Gozo EG, Cosnow I, Cohen HC et al. The heart in sarcoidosis. Chest 1971; 60: 379-388

${ }^{5}$ Sharma OP, Maheshwari A, Thaker K. Myocardial sarcoidosis. Chest 1993; 103: $253-258$

${ }^{6}$ Fleming H. Cardiac Sarcoidosis. In: James D ed. Sarcoidosis and other granulomatous disorders. New York: Marcel Dekker, 1994: 323-334

${ }^{7}$ Shammas RL, Movahed A. Sarcoidosis of the heart. Clin Cardiol 1993; 16: $462-472$

${ }^{8}$ Kirsten D, Schaedel H. Herzbeteiligung bei Lungensarkoidose. Z Erkrank Atm Org 1984; 162: 99-107

${ }^{9}$ Kirsten D, Schaedel H, Kessler G. Retrospektive Auswertung des Sarkoidosekrankengutes der Jahrgänge 1970-1979 der Zentralklinik für Herz- und Lungenkrankheiten in Bad Berka zur Auffindung möglicher Herzbeteiligung. Z Erkrank Atm Org 1984; 162: 108-117

${ }^{10}$ Kirsten D. Sarkoidose in Deutschland. Pneumologie 1995; 49: $378-382$

${ }^{11}$ Kirsten D. Herzsarkoidose. Pneumologie 1994; 48: 737- 743

12 Sharma OP. Diagnosis of cardiac sarcoidosis: an imperfect science, a hesitant art. Chest 2003; 123: $18-19$

${ }^{13}$ Flemming HA. Sarcoid heart disease. Br Heart J 1974; 36: 54-68

${ }^{14}$ Johns CJ, Michele TM. The clinical management of sarcoidosis: a 50 year experience at the John Hopkins Hospital. Medicine 1999; 78 : $65-111$

${ }^{15}$ Newman LS, Rose CS, Maier LA. Sarcoidosis. N Engl J Med 1997; 336: $1224-1234$

${ }^{16}$ Scadding JG. Sarcoidosis. London: Eyre and Spottiswoode, 1967: 291

17 American Thoracic Society . Statement on sarcoidosis. Am J Respir Crit Care Med 1999; 160: 736-755

${ }^{18}$ Lynch JP, Sharma OP, Baughman RP. Extrapulmonary sarcoidosis. Semin Respir Infect 1998; 13: 229-254

${ }^{19}$ Duke C, Rosenthal E. Sudden death caused by cardiac sarcoidosis in childhood. J Cardiovasc Electrophysiol 2002; 13: 939-942

${ }^{20}$ Yigla M, Badarna-Abu-Ria N, Tov N et al. Sarcoidosis in northern Israel: clinical characteristics of 120 patients. Sarcoidosis Vasc Diffuse Lung Dis 2002; 19: 220-226

${ }^{21}$ Schaedel H, Kisten D, Schmidt $\mathrm{H}$ et al. Sarcoid heart disease results of follow up investigations. Eur Heart J 1991; 12: 26-27

22 Uemura A, Morimoto S. Cardiac sarcoidosis. Nippon Rinsho 2002; 60: $1794-1800$

${ }^{23}$ Arunabh S, Verma N, Brady TM. Massive pericardial effusion in sarcoidosis. Am Fam Physician 1998; 58: 660-662

${ }^{24}$ Kinney EL, Jackson GL, Reeves WC et al. Thallium scan myocardial defects and echocardiographic abnormalities in patients with sarcoidosis without clinical cardiac dysfunction : an analysis of 44 patients . Am J Med 1980; 68: 497-503

25 Tellier P, Paycha F, Antony I et al. Reversibility by dipyridamole of thallium-201 myocardial scan defects in patients with sarcoidosis. Am J Med 1988; 85: 189- 193

${ }^{26}$ Fahy GJ, Marwick T, McCreery CJ et al. Doppler echocardiographic detection of the left ventricular diastolic dysfunction in patients with pulmonary sarcoidosis. Chest 1996; 109: $62-66$
${ }^{27}$ Burstow DJ, Tajik AJ, Bailey KR et al. Two-dimensional echocardiographic findings in systemic sarcoidosis. Am J Cardiol 1989; 63: $478-482$

${ }^{28}$ Buckley BH, Rouleau JR, Whitaker JQ et al. The use of thallium for myocardial perfusion imaging in sarcoid heart disease. Chest 1977; 72: $27-32$

${ }^{29}$ Roberts WC, McAllister Jr HA, Ferrans VJ. Sarcoidosis of the heart: a clinicopathologic study of 35 necropsy patients (group1) and review of 78 previously described necropsy patients (group 2). Am J Med 1977; 63: 86-108

30 Silverman KJ, Hutchins GM, Buckley BH. Cardiac Sarcoid: a clinicopathologic study of 84 unselected patients with systemic sarcoidosis Circulation 1978; 58: 1204-1211

${ }^{31}$ Matsui Y, Iwai K, Tachibana T et al. Clinicopathological study on fatal myocardial sarcoidosis. Ann NY Acad Sci 1976; 278: 455-469

32 Longscope WT, Freiman DG. A study of sarcoidosis: based on a combined investigation on 160 cases, including 30 autopsies from John Hopkins Hospital and Massachusetts General Hospital. Medicine 1952; 3: 1- 152

33 Perry A, Vuitch F. Causes of death in patients with sarcoidosis: a morphologic study of 38 autopsies with clinicopathologic correlations. Arch Pathol Lab Med 1995; 119: 167-172

${ }^{34}$ Iwai K, Skiguchi M, Hosoda Yet al. Racial differences in cardiac sarcoidosis noted at autopsy. Sarcoidosis 1994; 11: $248-251$

35 Gideon NM, Mannino DM. sarcoidosis mortality in the United States 1979-1991: an analysis of multiple cause mortality data. Am J Med 1996; 100: $423-427$

${ }^{36}$ Virmani R, Bures JC, Roberts WC. Cardiac sarcoidosis: a major cause of sudden death in young individuals. Chest 1980; 77: 423-428

${ }^{37}$ Fleming HA. Sarcoid heart disease. Br Med J 1973; 1: 174- 175

${ }^{38}$ Sekiguchi M, Numao Y, Imai M et al. Clinical and histopathological profile of sarcoidosis of the heart and acute idiopathic myocarditis . concepts through a study employing endomyocardial biopsy. I: Sarcoidosis. Jpn Circ J 1980; 44: 249-263

${ }^{39}$ Sekiguchi M, Yazaki Y, Isobe M et al. Cardiac sarcoidosis: diagnostic, prognostic and therapeutic considerations. Cardiovasc Drugs Ther 1996; 10: 495-510

${ }^{40}$ Tachibana T, Ohmori F, Ueda E. Clinical study on cardiac sarcoidosis. Ann NY Acad Sci 1986; 465: 530-542

${ }^{41}$ Stewart RE, Graham DM, Godfrey GW et al. Rapidly progressive heart failure resulting from cardiac sarcoidosis. Am Heart J 1988; 115: $1324-1326$

42 Yazaki Y, Isobe M, Hiroe $M$ et al. Prognostic determinants of long term survival in Japanese patients with cardiac sarcoidosis treated with prednisolone. Am J Cardiol 2001; 88: 1006-1010

${ }^{43}$ Israel RH, Poe RH. Massive pericardial effusion in sarcoidosis. Respiration 1994; 61: 176-180

${ }^{44}$ Garret J, O'Neill H, Blake S. Constrictive pericarditis associated with sarcoidosis. Am Heart J 1984; 107: 394- 397

${ }^{45}$ Yazaki Y, Hongo M, Hiroyochi Y et al. Cardiac sarcoidosis in Japan: treatment and prognosis. In: Sekiguchi M, Richardson PJ. Prognosis and treatment of cardiomyopathy and myocarditis. Tokyo: Univ of Tokyo Press, 1994: 351 - 353

46 Zoneraich S, Gupta MP, Metha J et al. Myocardial sarcoidosis presenting as acute mitral insufficiency. Chest 1974; 66: $452-454$

${ }^{47}$ Numao Y, Sekiguchi M, Fruie T et al. A study of cardiac involvement in 963 cases of sarcoidosis by ECG and endomyocardial biopsy. Ann NY Acad Sci 1976; 76: 607-614

${ }^{48}$ Gibbons WJ, Levy RD, Nava S et al. Subclinical cardiac dysfunction in sarcoidosis. Chest 1991; 100: 44-50

${ }^{49}$ Suzuki T, Kanda T, Kubota $S$ et al. Holter monitoring as a noninvasive indicator of cardiac involvement in sarcoidosis. Chest 1994; 106: $1021-1024$

${ }^{50}$ Thunell M, Bjerle P, Karp K et al. Exercise test in patients with sarcoidosis: the importance of repolarization disturbances. Acta Med Scand 1988; 223: 69-73

${ }^{51}$ Larsen F, Pehrsson SK, Hammar N et al. ECG-abnormalities in Japanese and Swedish patients with sarcoidosis. A Comparison. Sarcoidosis Vasc diffuse Lung Dis 2001; 18: $284-288$

52 Delobbe A, Perault H, Maitre J et al. Impaired exercise response in sarcoid patients with normal pulmonary function. Sarcoidosis Vasc Diffuse Lung Dis 2002; 19: 148-153

53 Kinney E, Murthy R, Ascunce G et al. Pericardial effusions in sarcoidosis. Chest 1979; 76: 476-478 
${ }^{54}$ Angomachalelis N, Hourzamanis A, Salem N et al. Pericardial effusion concomitant with specific heart musle disease in systemic sarcoidosis. Postgrad Med J 1994; 70: S8 -S12

${ }^{55}$ Shiff AD, Blatt CJ, Colp C. Recurrent pericardial effusion secondary to sarcoidosis of the pericardium.

${ }^{56}$ Lewin RF, Mor R, Spitzer S et al. Echocardiographic evaluation of patients with systemic sarcoidosis. Am Heart J 1985; 110: 116-122

${ }^{57}$ Gregor P, Widimsky P, Sladkova Tet al. Echocardiography in sarcoidosis. Jpn Heart J 1984; 25: 499-508

${ }^{58}$ Friart A, Philippart C, Bruart J. Echocardiography in systemic sarcoidosis. Lancet 1987; 1: 513

${ }^{59}$ Moiseyev SV, Kornev BM, Shatkovsky NP et al. Noninvasive diagnosis of cardiac sarcoidosis. Lancet 1987; 2: 739-740

${ }^{60}$ Winter SL, Cohen M, Grennberg S et al. Sustained ventricular tachycardia associated with sarcoidosis: assessment of the underlying cardiac anatomy and the prospective utility of programmed ventricular stimulation, drug therapy and implantable anti-tachycardia device. J Am Coll Cardial 1997; 18: 937 -943

${ }^{61}$ Le Guludec D, Menad F, Faraggi M et al. Myocardial sarcoidosis: clinical value of technetium-99m-sestamibi tomo-scintigraphy. Chest 1994; 106: 1675-1682

62 Tellier P, Valeyre D, Nitenberg A et al. Cardiac sarcoidosis: reversion of myocardial perfusion abnormalities by dipyridamole. Eur J Nucl Med 1985; 11: $201-204$

63 Yamamoto N, Gotoh K, Yagi Y et al. Thallium-201-myocardial SPECT findings at rest in sarcoidosis. Ann Nucl Med 1993; 7: 97-103

${ }^{64}$ Valeyre D, Marsac J, Gambini G et al. Assessment of sarcoid heart disease: a study of 37 patients with thoracic sarcoidosis. In: Chretien J, Marsac J, Saltiel JC (eds.). Sarcoidosis and other granulomatous diseases. Oxford: Pergamon, 1983: 296-300

${ }^{65}$ Haywood LJ, Sharma OP, Siegel ME et al. Detection of myocardial sarcoidosis by thallium201 imaging. J Natl Med Assoc 1982; 74: 959-964

${ }^{66}$ Fields CL, Ossorio MA, Roy TM et al. Thallium-201 scintigraphy in the diagnosis and management of myocardial sarcoidosis. South Med J 1990; 83: 339-342

67 Okayama K, Kurata C, Tawarahara K et al. Diagnostic and prognostic Value of myocardial scintigraphy with thallium-201 and gallium-67 in cardiac sarcoidosis. Chest 1995; 107: 330-334

${ }^{68}$ Mana J. Nuclear imaging: 67 Gallium, 201 thallium, 18F-labelled fluoro-2-deoxy-D-glucose positron emission tomography. Clin Chest Med 1997; 18: 799-811

${ }^{69}$ Nitenberg A, Foult JM, Blanchet F et al. Multifactorial determinants of reduced coronary flow reserve in after dipyridamole in dilated cardiomyopathy. Am J Cardiol 1985; 55: 748 - 754

70 Shimada T, Shimada K, Sakane T et al. Diagnosis of cardiac sarcoidosis and the evaluation of the effect of steroid therapy by Gadolinium DTPA-enhanced magnetic resonance imaging. Am J Med 2001; 110: $520-527$

71 Skold CM, Larsen FF, Rasmussen E et al. Determination of cardiac involvement in sarcoidosis by magnetic resonance imaging and Doppler echocardiography. J Intern Med 2002; 252 (5): 465-471

72 Vignaux O, Dhote R, Duboc D et al. Clinical significance of myocardial magnetic resonance abnormalities in patients with sarcoidosis: a 1-year follow up study. Chest 2002; 122: 1895-1901

${ }^{73}$ Tajima T, Naito T, Dohi Y et al. Ga-67 and TI-201 imaging in sarcoidosis involving myocardium. Clin Nucl Med 1981; 6: 120-121

${ }^{74}$ Nagatomo Y, Imamura T, Koiwaya Y et al. Thallium-201 and gallium-67 myocardial scintigraphy for the evaluation of sarcoid involvement. Clin Nucl Med 1987; 12: 335-336

${ }^{75}$ Hirose Y, Ishida Y, Hayashida K et al. Myocardial involvement in patients with sarcoidosis: an analysis of 75 patients. Clin Nucl Med 1994; 19: $522-526$

${ }^{76}$ Kurata C, Sakata K, Taguchi T et al. SPECT imaging with TI-201 and Ga -67 in myocardial sarcoidosis. Clin Nucl Med 1990; 15: 408 - 411

77 Taki J, Nakajima K, Bunko H et al. Cardiac sarcoidosis demonstrated by TI-201 and Ga-67 SPECT imaging. Clin Nucl Med 1990; 15: $636-639$

${ }^{78}$ Tawarahara K, Kurata C, Okayama K et al. Thallium-201 and gallium-67 single photon emission computed tomographic imaging in cardiac sarcoidosis. Am Heart J 1992; 124: 1383-1384

79 Riedy K, Fisher MR, Belic N et al. MR imaging of myocardial sarcoidosis. Am J Roentgenol 1988; 151: 915 - 916
${ }^{80}$ O'Connell JB, Henkin RE, Robinson JA et al. Gallium-67 imaging in patients with dilated cardiomyopathy and biopsy-proven myocarditis. Circulation 1984; 70: 58-62

${ }^{81}$ Forman MB, Sandler MP, Sacks GA et al. Radionuclide imaging in myocardial sarcoidosis : demonstration of myocardial uptake of technetium $99 \mathrm{~m}$ pyrophosphate and gallium. Chest 1983; 83: 578-580

${ }^{82}$ Angomachalellis N. Radionuclide imaging of specific heart musle disease in systemic sarcoidosis. Sarcoidosis 1992; 9: 245-253

${ }^{83}$ Eguchi M, Tsuchihashi K, Hotta D et al. Technetium $99 \mathrm{~m}$ sestamibi/ tetrofosmin myocardial perfusion scannino in cardiac and noncardiac sarcoidosis. Cardiology 2000; 94: 193 - 199

${ }^{84}$ Nishijima K, Miyahara Y, Furukawa K et al. Simultaneous assessment of right ventricular function and hypertrophy by Tc-99 m MIBI. Clin Nucl Med 1999; 24: 151 - 155

${ }^{85}$ Doherty MJ, Kumar SK, Nicholson AA et al. Cardiac sarcoidosis: the value of magnetic resonance imaging in diagnosis and assessment of response to treatment. Respir Med 1998; 92: 697-699

${ }^{86}$ Eliasch H, Juhlin-Dannfelt A, Sjogren I et al. Magnetic resonance imaging as an aid to the diagnosis and treatment evaluation of suspected myocardial sarcoidosis in a fighter pilot. Aviat Space Environ Med 1995; 66: 1010-1013

${ }^{87}$ Matsuki M, Matsuo M. MR findings of myocardial sarcoidosis. Clin Radiol 2000; 55: $323-325$

${ }^{88}$ Dupis JM, Victor J, Furber A et al. Magnetic resonance imaging in cardiac sarcoidosis: a case report. Arch Mal Coeur 1994; 87: 105-110

${ }^{89}$ Chandra M, Silverman ME, Oshinski J et al. Diagnosis of cardiac sarcoidosis aided by MRI. Chest 1996; 110: $562-565$

90 Bajaj AK, Kopelman HA, Echt DS. Cardiac sarcoidosis with sudden death: treatment with the automatic implantable cardioverter defibrillator. Am Heart J 1988; 116: 557-560

${ }^{91}$ Danias PG. Gadolinium-enhanced cardiac magnetic resonance imaging: expanding the spectrum of clinical applications. Am J Med 2001; 110: $591-592$

${ }^{92}$ Rieker O, Mohrs O, Oberholzer K et al. Cardiac MRI in suspected Myocarditis. Rofo Fortschr Geb Roentgenstr Neuen Bildgeb Verfahr 2002; 174: $1530-1536$

93 Vignaux O, Dhote R, Duboc D et al. Detection of myocardial involvement in patients with sarcoidosis applying T2-weighted, contrast-enhanced, and cine magnetic resonance imaging: initial results of a prospective study. J Comput Assist Tomogr 2002; 26: 762 - 767

${ }^{94}$ Mahrholdt H, Wagner A, Geissler A et al. Infarkt- und Vitalitätsdiagnostik mittels Kontrast-verstärkter Magnetresonanztomografie. Dtsch Med Wochenschr 2002; 127: 1264 - 1271

${ }^{95}$ Takedo N, Yokoyama I, Hiroe Y et al. Positron emission tomography predicted recovery of complete A-V nodal dysfunction in a patient with cardiac sarcoidosis. Circulation 2002; 105: 1144- 1145

${ }^{96}$ Okumura W, Iwasaki T, Ueda T et al. Usefulness of 18F-FDG PET for diagnosis of cardiac sarcoidosis. Kaku Igaku 1999; 36: 341 - 348

${ }^{97}$ Ishikawa T, Kondoh H, Nakagawa S et al. Steroid therapy in cardiac sarcoidosis: increased left ventricular contractility concomitant with electrocardiographic improvement after prednisolone. Chest 1984; 85: $445-447$

${ }^{98}$ Lorell B, Alderman EL, Mason JW. Cardiac sarcoidosis: diagnosis with endomyocardial biopsy and treatment with corticosteroids. Am J Cardiol 1978; 42: $143-146$

${ }^{99}$ Uemura A, Morimoto S, Hiramitsu S et al. Histologic diagnostic rate of cardiac sarcoidosis. Evaluation of endomyocardial biopsies. Am Heart J 1999; 138: 299-302

100 Ratner SJ, Fenoglio JJ, Ursel P. Utility of endomyocardial biopsy in the diagnosis of cardiac sarcoidosis. Chest 1986; 90: 528-533

101 Yazaki Y, Isobe M, Hiramitsu S et al. Comparison of clinical features and prognosis of cardiac sarcoidosis and idiopathic dilated cardiomyopathy. Am J Cardiol 1998; 82: 537 - 540

102 Takada K, Ina Y, Yamamoto M et al. Prognosis after pacemaker implantation in cardiac sarcoidosis in Japan: clinical evaluation of corticosteroid therapy. Sarcoidosis 1994; 11: 113-117

103 Baughman RP, Sharma OP, Lynch JPIII. Sarcoidosis: is therapy effective? Semin Respir Infect 1998; 13: 255-273

${ }^{104}$ Hiyama K. Corticosteroid therapy for sarcoidosis. Nippon Rinsho 2002; 60: $1827-1833$

105 Konishi M, Yoneda K, Takahashi K et al. Non-steroid therapy for sarcoidosis. Nippon Rinsho 2002; 60: 1834-1838

106 Baughman RP, Ohmichi M, Lower EE. Combination therapy for sarcoidosis. Sarcoidosis Vasc Diffuse Lung Dis 2001; 18: 133-137 
${ }^{107}$ Lynch JP, McCune WJ. Immunosuppressive and cytotoxic pharmacotherapy for pulmonary disorders. Am J Respir Crit Care Med 1997; 155: $395-420$

108 Zic JA, Horowitz DH, Arzubiaga C et al. Treatment of cutaneous sarcoidosis with chloroquine: review of the literature. Arch Dermatol 1991; 127: $1034-1040$

109 Baltzan M, Mehta S, Kirkham TH et al. Randomized trial of prolonged chloroquine therapy in advanced pulmonary sarcoidosis. Am J Respir Crit Care Med 1999; 160: $192-197$

110 Adams JS, Diz MM, Sharma OP. Effective reduction in the serum 1,25-dihydroxyvitamine $\mathrm{D}$ and Calcium concentration in sarcoidosisassociated hypercalcemia with short course chloroquine therapy. Ann Intern Med 1989; 111: 437 - 438

111 Sharma OP. Neurosarcoidosis: a personal perspective based on the study of 38 patients. Chest 1997; 112: 220-228

112 Kaye O, Pallazo E, Grossin M et al. Low dose methotrexate: an effective corticosteroid sparing-sparing agent in the musculoskeletal manifestations of sarcoidosis. Br J Rheumatol 1995; 34: 642 - 644

113 Webster GF, Razsi LK, Sanchez M et al. Weekly low-dose methotrexate therapy for cutaneous sarcoidosis. J Am Acad Dermatol 1991; 24: $451-454$

114 Baughman RP, Lower EE. A clinical approach to the use of methotrexate for sarcoidosis. Thorax 1999; 54: $742-746$

115 Müller-Quernheim J, Kienast K, Held M et al. Treatment of chronic sarcoidosis with an azathioprine/prednisolone regimen. Eur Resp J 1999; 14: $1117-1122$

116 Agbogu BN, Stern BJ, Sewell C et al. Therapeutic considerations in patients with refractory neurosarcoidosis. Arch Neurol 1995; 52: $875-879$

117 Lower EE, Broderick JP, Brott TG et al. Diagnosis and management of neurological sarcoidosis. Arch Intern Med 1997; 157: 1864- 1868

118 Demeter SL. Myocardial sarcoidosis unresponsive to steroids: treatment with cyclophosphamide. Chest 1988; 94: 202-203

119 Stern BJ, Schonfeld SA, Sewell C et al. The treatment of neurosarcoidosis with cyclosporine. Arch Neurol 1992; 49: 1065 - 1072

${ }^{120}$ Martinet Y, Pinkston P, Saltini C et al. Evaluation of the in vitro and in vivo effects of cyclosporine on the lung T-lymphocyte alveolitis on active pulmonary sarcoidosis. Am Rev Respir Dis 1988; 138: $1242-1248$

${ }^{121}$ Wyser CP, Schalkwyk EM van, Alheit B et al. Treatment of progressive pulmonary sarcoidosis with cyclosporin A: a randomized controlled trial. Am J Respir Crit Care Med 1997; 156: 1371-1376

122 Carlesimo M, Giustini S, Rossi A et al. Treatment of cutaneous and pulmonary sarcoidosis with thalidomide. J Am Acad Dermatol 1995; 32: $866-869$

123 Baughman RP, Judson MA, Teirstein AS et al. Thalidomide for chronic sarcoidosis. Chest 2002; 122: 227-232
124 Zabel P, Entzian P, Dalhoff K et al. Pentoxifylline in the treatment of sarcoidosis. Am J Respir Crit Care Med 1997; 155: 1665 - 1669

${ }^{125}$ Baughman RP. Infliximab for refractory sarcoidosis. Sarcoidosis Vasc Diffuse Lung Dis 2001; 18: 70 - 74

${ }^{126}$ Yee AM, Pochapin MB. Treatment of complicated sarcoidosis with infliximab anti-tumor necrosis factor-alpha therapy. Ann Intern Med 2001; 135: 27-31

${ }^{127}$ Mezaki T, Chinushi M, Washizuka T et al. Discrepancy between inducibility of ventricular tachycardia and activity of cardiac sarcoidosis: requirement of defibrillator implantation for the inactive stage of cardiac sarcoidosis. Intern Med 2001; 40: 731 - 735

${ }^{128}$ Huang PL, Brooks R, Carpenter C et al. Anti-arrhythmic therapy guided by programmed electrical stimulation in cardiac sarcoidosis with ventricular tachycardia. Am Heart J 1991; 121: 599-601

129 Kowey PR, Waxman HL, Greenspon A et al. Value of electro physiologic testing in patients with previous myocardial infarction and nonsustained ventricular tachycardia. Philadelphia Arrhythmia Group. Am J Cardiol 1990; 65: 594-598

${ }^{130}$ Poll DS, Marchlinski FE, Buxton AEet el. Usefullness of programmed stimulation in idiopathic dilated cardiomyopathy. Am J Cardiol 1986; 58: $992-997$

${ }^{131}$ Hsia HH, Marchlinski FE. Electrophysiology studies in patients with dilated cardiomyopathy. Card Electrophysiol Rev 2002; 6: 472 - 481

132 Huikuri HV, Castellanos A, Myersburg RJ. Sudden death due to cardiac arrhythmias. N Engl J Med 2001; 345: 1473 - 1482

133 Paz HL, McCormick DJ, Kutalek SP et al. The automated implantable cardiac defibrillator: prophylaxis in cardiac sarcoidosis. Chest 1994; 106: 1603 - 1607

134 Oni AA, Hershberger RE, Norman DJ et al. Recurrence of sarcoidosis in a cardiac allograft: control with augmented corticosteroids. J Heart Lung Transplant 1992; 11: 367-369

${ }^{135}$ Martinez FJ, Orens JB, Deeb M et al. Recurrence of sarcoidosis following bilateral allogenic lung transplantation. Chest 1994; 106: 1597-1599

${ }^{136}$ Valantine HA, Tazelaar HD, Macoviak J et al. Cardiac sarcoidosis: response to steroids and transplantation. J Heart Transplant 1987; 6: $244-250$

137 Shorr AF, Davies DB, Nathan SD. Outcomes for patients with sarcoidosis awaiting lung transplantation. Chest 2002; 122: 233-238

138 Meyer A, Schafer H, Doring V et al. Heart transplantation in myocardial sarcoidosis. Studies on the explanted heart.Dtsch Med Wochenschr 1999; 124: 1131 - 1134

${ }^{139}$ Angomachalelis N, Hourzamanis A, Vamvalis C et al. Doppler echocardiographic evaluation of left ventricular diastolic function in patients with systemic sarcoidosis. Postgrad Med J 1992; 68: 52 - 56 